\title{
Intégration des TIC par les enseignants : premiers résultats d'une enquête suisse
}

\section{Stéphanie Heer}

Haute École Pédagogique Berne-Jura-Neuchâtel, SUISSE

stephanie.heer@hep-bejune.ch

\section{Abdeljalil Akkari}

Haute École Pédagogique Berne-Jura-Neuchâtel, SUISSE

akkari.abdeljalil@hep-bejune.ch

\section{Réflexion pédagogique}

\section{Résumé}

Cet article propose tout d'abord un état de la littérature scientifique dans les domaines de l'intégration et de la formation aux TIC dans la pratique enseignante. Dans une deuxième partie sont présentés les premiers résultats d'une enquête menée sur l'intégration des TIC dans le système scolaire du canton du Jura en Suisse. L'article se termine par la présentation des premières conclusions de cette recherche et une discussion sur les opportunités pédagogiques qu'ouvrent les TIC pour les enseignants débutants.

\section{Summary}

In the first part, this paper summarizes the existing scientific literature on the use of information and communication technologies (ICT) by teachers. In the second part, results of data analysis from a regional survey of the Swiss educational ICT infrastructure and its use in schools are presented. In the third part, we discuss the opportunity for beginning teachers to transform their pedagogical practices with the increasing availability of ICT in school. 
Dans le contexte d'une étude menée par le secteur recherche de la Haute École Pédagogique Berne-Jura-Neuchâtel en collaboration avec le service de l'enseignement du canton du Jura, nous avons réalisé une enquête sur l'intégration des technologies de l'information et de la communication (TIC) par les enseignants du canton du Jura, tous niveaux confondus. Cette étude avait deux objectifs: d'une part, évaluer $l^{\prime}$ impact du programme EDUC $2006^{1}$ en observant le degré d'intégration des TIC et, d'autre part, réguler l'introduction des TIC en proposant des pistes concrètes ou des recommandations pour améliorer la qualité des formations initiale et continue, et favoriser la pérennité et l'efficacité pédagogiques du programme.

L'article est subdivisé en deux parties: la première fait état de la littérature scientifique francophone et anglophone dans les domaines de l'intégration et de la formation aux TIC dans la pratique enseignante; la seconde présente notre recherche proprement dite, exposant successivement la méthodologie, les principaux résultats ainsi que leur analyse.

\section{La littérature scientifique}

Nous vivons actuellement une époque de rapides changements technologiques. Les TIC font désormais partie intégrante de notre société, dans un contexte tant professionnel que privé. Le développement rapide des TIC nous oblige donc à former aussi bien les enseignants que les élèves à ces nouvelles compétences. Or, il apparaît que l'adaptation aux usages des TIC en classe est trop lente. Selon Bibeau (1999, p.2), «si le système scolaire offre si peu de performance, c'est peutêtre qu'il constitue l'un des derniers bastions où les technologies de l'information et de la communication (TIC) n'ont pas été encore véritablement intégrées ». De plus en plus d'écoles se voient dotées de nouveaux outils technologiques. Cependant, un nombre important d'enseignants se sentent démunis ou à l'écart face à ce nouveau matériel.

\section{Adaptation aux usages des TIC en classe}

Dans un premier temps, il semble important de se demander comment s'intègrent les TIC en classe. Est-ce que cette intégration demande une modification des pratiques d'enseignement? Larose, Grenon et Lafrance (1999, p. 23) postulent que :

«les recherches sur l'intégration des TIC dans l'enseignement identifient deux courants majeurs. Le premier, fondé sur une épistémologie socioconstructiviste, analyse cette intégration comme vitale et favorable à la modification des pratiques d'enseignement. Le deuxième, de type néocomportementaliste et pragmatique, considère les TIC comme de simples outils compatibles avec un enseignement traditionnel. »

Selon l'OCDE (1998), pour une prise en compte tangible des TIC dans le secteur de l'éducation, il ne suffit pas de combiner l'utilisation de l'outil informatique avec les pédagogies existantes mais il est pertinent d'adapter l'enseignement aux nouvelles possibilités qui s'offrent.

D'autres spécialistes insistent sur la nécessité de profiter de la valeur ajoutée pédagogique rendue possible avec l'usage des TIC pour permettre une amélioration de la pédagogie et du rapport au savoir (Perrenoud,1998; Peraya, 2002; Karsenti, Peraya et Viens, 2002a).

À travers l'analyse historique de l'introduction des TIC à l'école, force est de constater que cette intégration en classe se situe entre deux approches institutionnelles. La première consiste à dire que les TIC peuvent être vues comme une 
discipline scolaire à part entière avec des compétences spécifiques en TIC qu'il faut maîtriser à la sortie de l'école. La seconde conception affirme que les TIC sont des outils transversaux qui devraient être intégrés dans toutes les disciplines scolaires et font partie intégrante de tout enseignement. Cette deuxième approche induirait une restructuration profonde de la manière d'enseigner les disciplines scolaires.

\section{Formation des enseignants aux usages des TIC}

Dans un deuxième temps, il est important de préciser qu'une intégration efficace des TIC ne peut se réaliser sans formation à l'appui. Former les enseignants à l'usage des TIC en classe semble important dans notre société où l'innovation technologique prend de plus en plus d'ampleur. Peu de recherches ont été réalisées au sujet de la formation des enseignants, car les chercheurs se sont concentrés prioritairement sur l'observation des avantages et inconvénients de l'intégration des TIC en classe en se focalisant sur les élèves. À l'heure actuelle, de plus en plus de recherches se proposent d'analyser la situation de la formation des enseignants et les facteurs influençant l'adaptation aux usages des TIC en classe. Larose, Lenoir, Karsenti et Grenon (2002) s'intéressent aux facteurs favorisant ou inhibant l'adaptation aux usages des TIC dans la pratique des enseignants, ainsi qu'à l'influence du modelage de la formation pratique sur la reproduction des modèles d'utilisation des TIC. Il semble ainsi important d'observer le transfert des compétences acquises en formation à la pratique enseignante. Plusieurs facteurs contextuels peuvent nuire à ce transfert, comme l'équipement dans la classe ou à l'école, le temps à disposition, la reconnaissance de l'institution, la formation des directeurs d'école, la collaboration entre collègues. Isabelle, Lapointe et Chiasson (2002) se sont intéressées au rôle des directeurs d'école dans l'insertion des TIC en classe. Elles observent que plus les directeurs d'école disent faire euxmêmes une utilisation pédagogique et technologique des TIC, plus il leur semble facile d'utiliser les méthodes pédagogiques renouvelées avec les $\mathrm{TIC}$, plus ils se sentent à l'aise de soutenir les enseignants dans l'intégration des TIC et plus ils ont une vision positive quant à l'avenir des TIC dans le système éducatif (p. 336).

Afin de permettre une meilleure implantation des TIC, Deaudelin, Dussault et Brodeur (2002, cités par Karsenti, Peraya et Viens, 2002a, p. 258) proposent de «favoriser le transfert, mettre l'accent sur les communautés d'apprenants, susciter chez les enseignants une réflexion sur leur apprentissage et leur pratique, enfin, intervenir autant sur les croyances que sur les pratiques ».

Un autre type de recherche s'intéresse au sentiment de compétence et d'anxiété relativement à l'utilisation des TIC. Selon Carugati et Tomasetto (2002), la formation peut jouer un rôle déterminant en ce qui concerne la réduction du facteur d'anxiété. Elle pourrait ainsi pousser les enseignants à une meilleure acceptation de l'innovation. Dès que l'ordinateur a pénétré dans la classe, la formation ne pouvait plus rester à l'écart des TIC. Les compétences acquises en formation devraient comprendre des compétences techniques, mais surtout pédagogiques. L'enseignant en formation doit être capable d'acquérir un esprit critique face à l'utilisation des TIC, réfléchir à des séquences d'enseignement utilisant les TIC et ainsi adapter son enseignement.

Comme le décrit Rogers (2000, cité par Larose et al., 2002, p. 269) :

«l'exposition des étudiantes et des étudiants aux discours et aux pratiques 
d'utilisation des TIC par les enseignantes

et les enseignants chevronnés et

l'interaction avec ces derniers semblent constituer un des facteurs affectant les probabilités de transfert des compétences informatiques construites en milieu universitaire sur le plan des pratiques professionnelles des novices. »

Il conclut en exprimant que «seules l'ampleur et la profondeur du changement de rapport au savoir ainsi qu'à la relation enseignementapprentissage peuvent être garantes de modifications significatives $\mathrm{du}$ rapport d'appropriation des outils didactiques par les enseignantes et enseignants » (p. 284). Coen (sous presse) ne dit pas autre chose en arguant que le défi actuel pour les enseignants consiste à transformer leur enseignement pour le rendre apte à intégrer les TIC davantage qu'intégrer les TIC dans leur enseignement traditionnel.

En d'autres termes, Carugati et Tomasetto (2002, p. 306) postulent que :

«les connaissances implicites et les attitudes des enseignantes et enseignants novices au regard du profil d'utilisation pédagogique des TIC dépendent donc en grande partie des apprentissages informels réalisés par observation plus ou moins systématique dans les milieux de la pratique. »

De plus, ils découvrent que :

«les enseignantes les plus expertes sont celles qui témoignent de moins d'anxiété, d'un désir plus élevé d'améliorer leur niveau d'expertise, de plus de confiance dans les NTIC, jusqu'au point d'en préconiser une utilisation systématique dans leurs classes (p. 321). »
Les résultats des différentes recherches montrent l'importance de l'observation et de l'imitation des pratiques pédagogiques ainsi que l'influence du contexte pour augmenter la fréquence d'utilisation des TIC en classe.

Charlier, Daele et Deschryver (2002, p. 350) proposent une approche intégrée de la formation aux TIC. Elles proposent une formation basée sur le concept de recherche-action-formation, qui permette aux enseignants en formation et aux formateurs de mener un projet d'action en collaboration. Ainsi, les formateurs épaulent les enseignants en formation qui transmettront leurs compétences à la pratique enseignante. Selon Charlier et al. (2002, p. 350), « la recherche aide à réguler la formation, qui elle-même sert de support à la recherche. La formation sert de support à l'action des enseignants en formation, celle-ci est analysée lors de la formation ». Les auteures présentent trois projets de rechercheaction-formation : le projet Learn-Nett; le projet Intégration et exploitation d'Internet en classe dans le domaine de l'apprentissage des sciences et le projet Form@Hetice. Les apprenants participent à l'apprentissage collaboratif et acquièrent ainsi des compétences transversales liées à la communication et à la collaboration à distance. Le réseau et le soutien des formateurs, du groupe d'enseignants et de son établissement sont des variables indispensables pour une intégration réussie des TIC en formation. Les communautés de pratique ou d'apprentissage sont ainsi mises en avant.

Une autre recherche (Breuleux, Erickson, Laferrière et Lamon, 2002) s'intéresse à l'établissement de communautés d'apprentissage en réseau pour l'intégration pédagogique des TIC en formation des maîtres. Elle observe «le déploiement de pratiques innovatrices au sein de communautés branchées en réseau dont l'objectif est le renouvellement de l'apprentissage de la pédagogie par la collaboration » (Breuleux et al., 
2002, p.418). Les auteurs ont montré que « l'intégration pédagogique des TIC peut enrichir les interactions entre les étudiants en formation initiale, les enseignants en poste et les universitaires ».

De plus, selon Linard (2002, p. 152) :

« au plan cognitif, l'hyperacteur doit s'engager dans des activités techniques et sociales complexes, naviguer sans perdre de vue ses buts dans des espaces mixtes infinis de réel et de virtuel, prendre des initiatives en situation $\mathrm{d}$ 'incertitude et s'adapter au stress des changements permanents. »

En outre, Haeuw (2002, p. 81) émet l'hypothèse que :

«c'est non pas la maîtrise technologique qui sera déterminante dans le changement, mais l'augmentation des compétences qui permettent de communiquer, coopérer, s'organiser, gérer, qui obligent à considérer celui qui apprend comme un acteur à part entière, dont l'engagement et l'action influent sur l'efficience du dispositif, et avec qui il faut donc coopérer. »

Une étude récente portant sur sept programmes de formation initiale considérés comme les plus performants aux État-Unis montrait que moins de $50 \%$ des enseignants diplômés se déclaraient bien préparés dans le domaine des TIC (DarlingHammond; 2006). Selon l'auteure, cela démontre que la formation aux TIC est l'un des enjeux majeurs des récentes réformes dans la formation des enseignants.

Une politique volontariste et précoce de promotion de l'usage des TIC par les enseignants en formation semble être l'une des caractéristiques de base des programmes de formation initiale les plus performants. Dans leur étude sur les programmes performants de formation des enseignants aux États-Unis, Mergendoller, Johnston, Rockman et Willis (1994) constatent que l'un des meilleurs programmes a intégré les TIC dans la formation 10 ans avant les autres. Les TIC semblent donc constituer un indice permettant de situer un programme de formation des enseignants.

L'irruption des TIC dans le champ de la formation initiale des enseignants intervient à un moment où la formation fait l'objet d'importantes réformes dans de nombreux pays. Ainsi, la fermeture des Écoles Normales et le lancement des Hautes Écoles Pédagogiques en Suisse à la fin des années 1990 ont totalement transformé la formation des enseignants dans le pays en y incluant en particulier la dimension recherche et la notion de compétences professionnelles. La question cruciale est de savoir quelle quantité de formation en TIC est nécessaire, mais aussi quelles modalités pédagogiques seront dispensées. Le concept de compétence, qui est difficilement opérationnel dans de nombreuses composantes de la formation initiale, trouve dans les TIC un intéressant terrain d'expérimentation. Dans le cadre du programme de formation intitulé «F3-MITIC ${ }^{2}$ », par exemple, qui concerne toute la Suisse et qui vise la formation de formateurs de formateurs d'enseignants, le programme propose de traiter les questions pédagogiques soulevées par l'intégration des TIC dans la construction du savoir et d'élaborer des scénarios pédagogiques.

Les résultats des différentes recherches réalisées sur l'adaptation et la formation des enseignants aux usages des TIC nous montrent que même si les écoles sont de plus en plus équipées, cela ne veut pas dire que leur utilisation de cet équipement est optimale. Il reste encore beaucoup à réaliser au niveau de la formation des 
enseignants. Par ailleurs, la problématique de la formation en TIC reste à clarifier, en particulier quant au type de formation le plus propice à l'adoption des TIC.

À partir de ces résultats, il nous semble pertinent de s'intéresser à un contexte particulier (le canton du Jura en Suisse) et d'obtenir des informations sur l'équipement, la fréquence et le type d'utilisation, ainsi que la formation reçue en TIC. Il s'avère que dans ce

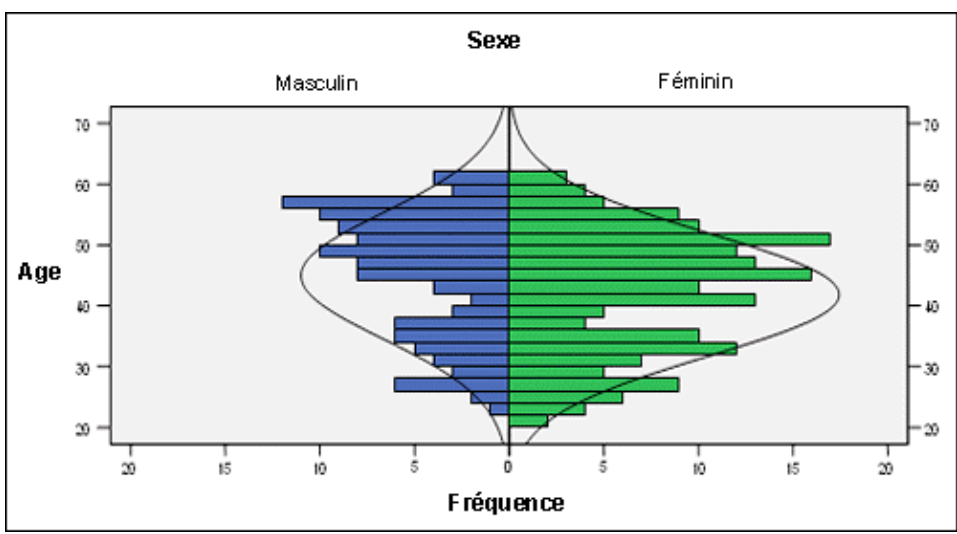

Graphique 1. Pyramide des âges et du sexe plus de $30 \%$. En effet, l'échantillon de notre étude est constitué de 298 enseignants formés majoritairement d'enseignants du primaire (55\%) suivis des enseignants du secondaire $(31 \%)$ et des enseignants du préscolaire (13\%). La pyramide ci-dessous vous montre le profil des âges et du sexe.

\section{Instrument}

Le questionnaire que nous avons envoyé à large échelle aux enseignants jurassiens se compose de différents thèmes: informations générales;

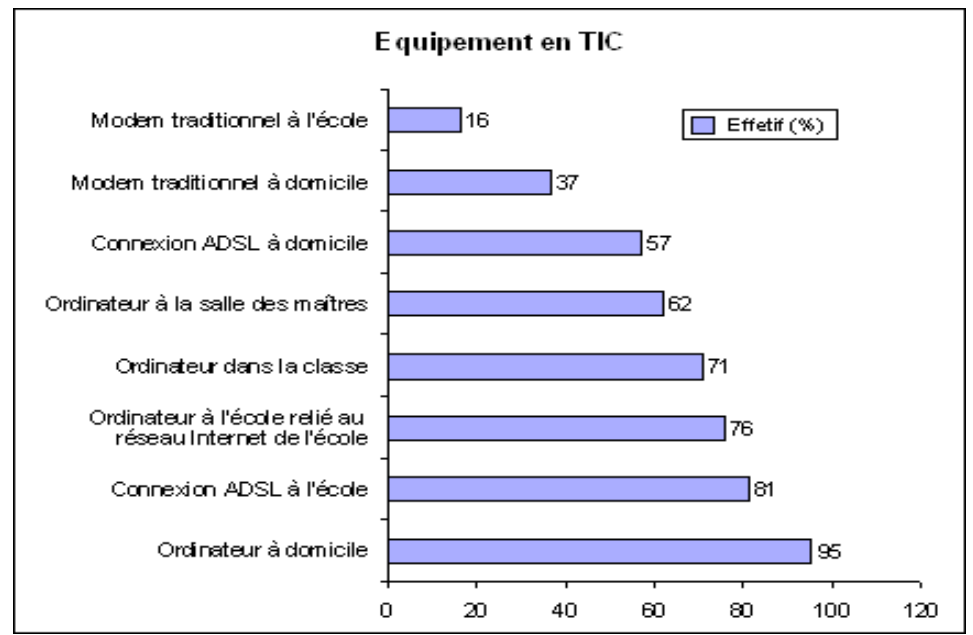

Graphique 2. Effectif des équipements en TIC

800 enseignants dont 310 de l'école secondaire, 350 de l'école primaire et 125 de l'école enfantine. Le taux de réponses aux questionnaires est de équipement, formation et connaissances informatiques; utilisation pédagogique des TIC et effets induits par les TIC. Nous nous sommes inspirés de différents travaux de recherche et plus particulièrement des travaux réalisés au sein du Groupe de recherche sur l'interdisciplinarité dans la formation à l'enseignement de l'Université de Sherbrooke (Larose et al., 1999; Larose et al., 2002; Larose, Grenon, Pearson, Morin et Lenoir, 2004).

Les principaux résultats de notre enquête, présentés ci-dessous, ne prennent pas en compte les représentations des enseignants. Nous avons préféré présenter dans cet article des 
données générales concernant l'équipement, la formation, la fréquence et le type de pratiques des TIC en classe.

\section{Principaux résultats}

\section{Équipement}

L'équipement en TIC joue un rôle important dans leur usage en classe. Si l'enseignant ne dispose pas d'un accès facilité à un matériel adéquat, il est probable qu'il sera moins enclin à faire l'effort d'utiliser les TIC avec ses élèves. Le graphique 2 ci-dessous montre que les enseignants jurassiens sont bien équipés en TIC. Seuls $10 \%$ ne disposent pas d'ordinateurs à domicile. Ils jouissent en majorité d'une connexion ADSL à l'école et sont reliés au réseau Internet de celle-ci. Ce sont avant tout les enseignants du primaire (156) qui ont au moins un ordinateur par classe. Seuls 38 enseignants du préscolaire et $15 \mathrm{du}$ secondaire ont la possibilité d'utiliser un ordinateur dans leur classe. N'oublions pas que ceux du secondaire ne disposent que rarement d'ordinateurs dans leur classe, ayant uniquement une salle d'informatique pour donner leur cours. En moyenne, les enseignants qui ont la possibilité d'avoir un ou plusieurs ordinateurs dans leur classe ont 1,9 ordinateur par classe, dont en moyenne 1,6 permet l'accès au courrier électronique ou à l'Internet. En outre, deux tiers des enseignants $(60 \%)$ pensent que leurs élèves n'utilisent pas d'autres ordinateurs en dehors des périodes d'enseignement. Précisons par ailleurs que $47 \%$ des enseignants du secondaire pensent que leurs élèves utilisent la salle d'informatique en dehors des cours. De plus, $87 \%$ des enseignants disent disposer de la salle d'informatique pour les cours.

\section{Formation}

Afin de motiver les enseignants à utiliser les TIC en classe, les formations initiale et continue jouent un rôle crucial. Or, on observe que relativement peu de participants à notre enquête ont été formés aux TIC durant la formation initiale à l'enseignement. Relevons que les enseignants formés aux TIC en formation initiale ont entre 25 et 30 ans (Graphique 3).

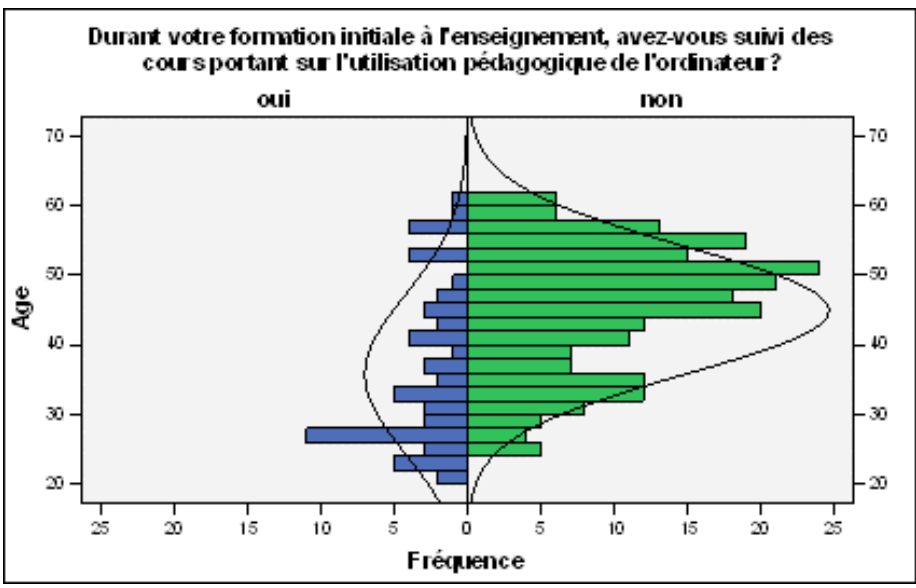

Graphique 3. Pyramide des âges en lien avec la formation initiale à l'utilisation édagogique de l'ordinateur
Malgré cela, nous n'observons pas de différence de fréquence d'utilisation entre les jeunes enseignants et les plus âgés qui ont bénéficié d'une formation initiale. Deux explications sont plausibles pour interpréter ces résultats qui peuvent paraître à première vue paradoxaux. D'une part, les enseignants novices ont des priorités autres que l'intégration des TIC lors de leur première insertion professionnelle. D'autre part, peu de programmes de formation initiale font des TIC une composante essentielle de leur curriculum.

Même si la majorité des enseignants ont eu la possibilité d'être formés par le biais de la formation continue, le fait que les novices ne se distinguent pas par un usage plus intensif des TIC devrait inciter les institutions de formation initiale à revoir la place des TIC dans les programmes. 
Fréquence et types d'utilisation

Les résultats nous indiquent que $56 \%$ des enseignants utilisent les TIC de 1 à 3 périodes par semaine et seulement $9 \%$ les utilisent plus de 4 périodes par semaine. De plus, il faut être attentif au fait que $27,5 \%$ ne font aucun usage des TIC. Ces résultats montrent que certains enseignants ne sont pas encore familiarisés avec l'usage des TIC en classe. Les enseignants jurassiens sont mieux équipés en TIC, mais les utilisent moins que la moyenne européenne. Cela montre de nouveau la nécessité d'ancrer l'usage des TIC dans la culture enseignante dès la formation initiale et de poursuivre cet effort dans la formation continue.

De plus, les résultats montrent qu'il n'y pas de différence de fréquence d'utilisation entre les enseignants débutants (entre 0 et 5 ans d'expérience) et les enseignants ayant plus de 5 années d'expérience. Parmi les enseignants débutants, $33 \%$ ne font aucune utilisation des TIC dans leur enseignement, $59 \%$ les utilisent 1 à 3 périodes par semaine et seuls $9 \%$ les utilisent plus de 3 périodes.

Finalement, en ce qui concerne les types de pratique des TIC dans l'enseignement (voir le graphique 4), les enseignants utilisent en premier lieu la recherche d'information dans le cadre de travaux spécifiques et les logiciels éducatifs dans le cadre d'interventions pédagogiques. Nous pouvons nuancer ces résultats par le niveau d'enseignement. Pour l'enseignement secondaire, le type d'utilisation prioritaire est la recherche d'informations sur Internet. Au primaire, il s'agit des logiciels éducatifs et au préscolaire, des logiciels de jeu.

Ces résultats soulèvent deux questions :

1. $28 \%$ des enseignants n'utilisent jamais les TIC alors qu'ils disposent d'un bon équipement et qu'ils ont reçu une formation initiale ou continue. Se pose alors la question de la qualité de la formation, de ses objectifs et de ses méthodes: celle-ci répond-elle réellement à la demande des enseignants?

2. Étant donné que les enseignants débutants ont reçu une formation initiale en TIC, nous nous attendions à ce qu'ils les utilisent davantage en classe et qu'ils motivent ainsi leurs collègues. Pourquoi les enseignants novices n'utilisent-ils pas davantage les TIC en classe? Est-ce que cela est dû au dispositif de formation initiale, à un manque de pratique des TIC durant les stages, à des questions liées à l'insertion professionnelle des enseignants ou à des problèmes organisationnels?

Ces premiers résultats montrent clairement que l'équipement en TIC, la formation, la fréquence et le type d'utilisation sont liés. Si une réforme éducative a pour objectif de développer l'usage des TIC pas les enseignants, alors tous ces éléments doivent être pensés simultanément.

\section{Discussion et suite de l'étude}

En tant que représentants de la nouvelle génération, les enseignants débutants devraient être plus à l'aise avec l'usage des TIC. Ils pourraient ainsi donner l'impulsion, mais surtout l'envie d'utiliser les TIC à leurs collègues. C'est ainsi que nous jugeons pertinent de nous intéresser à la formation initiale en particulier. Dans ce contexte, nous approfondirons dans une phase ultérieure l'analyse de la formation initiale des enseignants et le transfert possible des compétences dans la pratique des débutants.

À ce sujet, plusieurs études montrent que les enseignants nouvellement formés intègrent peu 


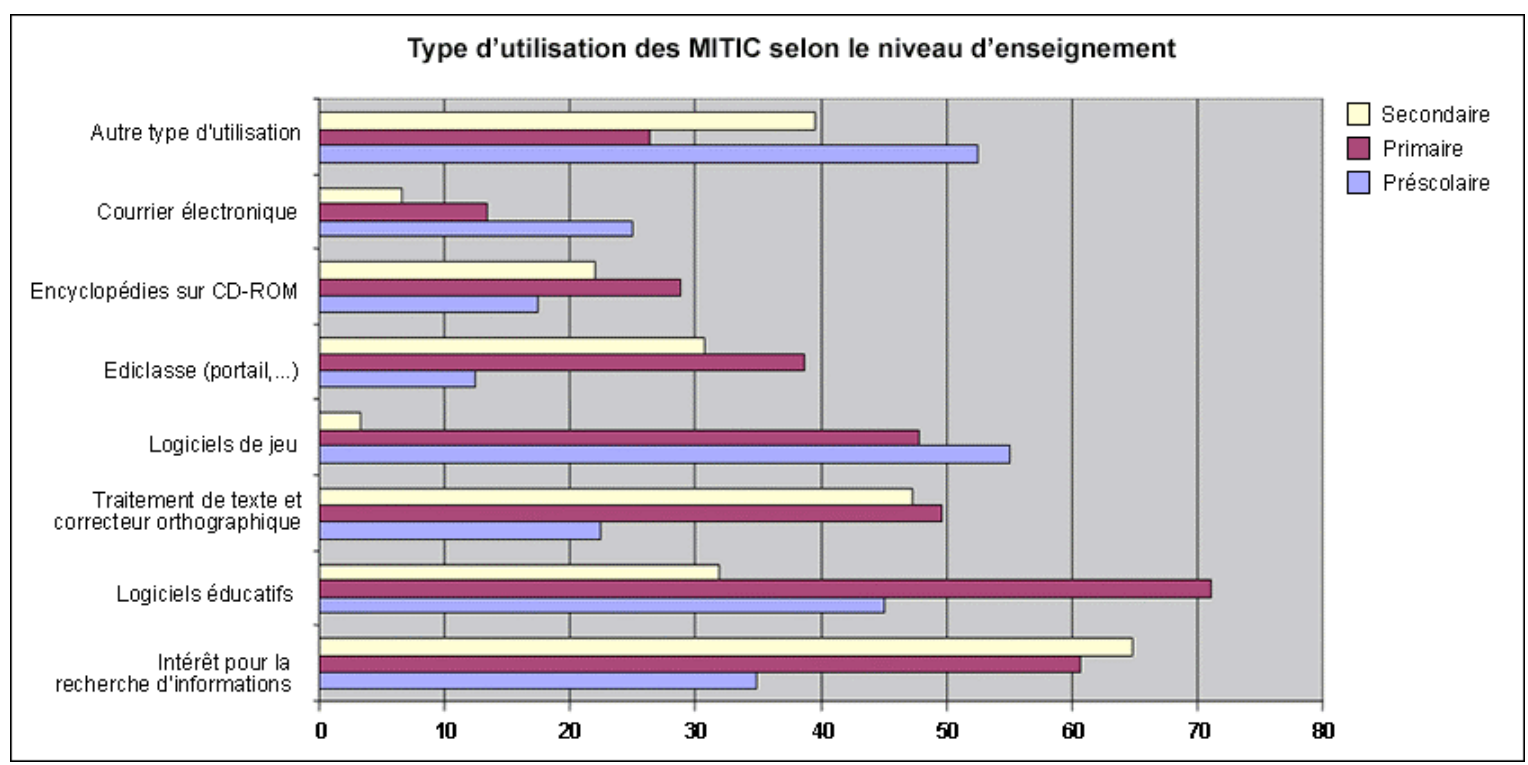

Graphique 4. Effectif des enseignants selon les types d'utilisation des MITIC dans l'enseignement et selon le niveau d'enseignement

les TIC en classe (Karsenti et Larose, 2005). De plus, la formation aux usages pédagogiques des TIC pose de nombreux écueils et obstacles. En particulier, le transfert de la théorie à la pratique professionnelle des enseignants reste problématique (Karsenti et Larose, 2005). Rogers (2000, cité par Larose et al., 2002, p. 269) décrit que :

«l'exposition des étudiantes et des étudiants aux discours et aux pratiques d'utilisation des TIC par les enseignantes et les enseignants chevronnés et l'interaction avec ces derniers semblent constituer un des facteurs affectant les probabilités de transfert des compétences informatiques construites en milieu universitaire sur le plan des pratiques professionnelles des novices. »

Ils concluent en exprimant que: «seules l'ampleur et la profondeur du changement de rapport au savoir ainsi qu'à la relation enseignement-apprentissage peuvent être garantes de modifications significatives $\mathrm{du}$ rapport d'appropriation des outils didactiques par les enseignantes et enseignants» (p. 284). Selon Larose et Peraya (2001, cités par Karsenti, Peraya et Viens, 2002b, p. 461), «la présence de modèles lors de la formation pourrait permettre aux futurs enseignants d'intégrer à leur tour les TIC, lorsqu'ils œuvreront auprès des élèves du primaire et du secondaire ». De plus, les auteurs précisent que «l'effet de modelage de ces contextes d'apprentissage sur l'utilisation des technologies et particulièrement sur les pratiques professionnelles des enseignants commence à être bien mis en évidence par un nombre croissant de recherches récentes ». Selon Carugati et Tomasetto (2002), la formation peut jouer un rôle déterminant en ce qui concerne la réduction du facteur d'anxiété et, en conséquence, elle le pourrait aussi sur une meilleure acceptation de l'innovation induite par les TIC. L'introduction des TIC dans les systèmes éducatifs s'accompagnera d'un questionnement permanent sur les effets attendus et potentiels de ces outils sur l'apprentissage et les relations pédagogiques entre élèves et enseignants. 
Il nous semble intéressant de partir de ces appuis théoriques pour observer, dans le contexte jurassien, la formation en TIC et le transfert possible dans la pratique. La Suisse apparaît comme l'un des pays développés où le niveau général d'équipement en TIC est satisfaisant. Cependant, le système scolaire helvétique ne brille pas par l'excellence de son niveau d'innovations pédagogiques basées sur les TIC. La recherche devrait nous fournir des éléments de réponse à cette situation assez paradoxale.

\section{Références}

Bibeau, R. (1999). L'élève rapaillé. Montréal : Université de Montréal [Page Web]. Récupéré du site http :/ / www. Robertbibeau.ca/rapail.html

Breuleux, A., Erickson, G., Laferrière, T. et Lamon, M. (2002). Devis sociotechniques pour l'établissement de communautés d'apprentissage en réseau pour l'intégration pédagogique des TIC en formation des maîtres. Revue des sciences de l'éducation, 28(2), p. 411-434.

Carugati, F. et Tomasetto, C. (2002). Le corps enseignant face aux technologies de l'information et de la communication dans les pratiques d'enseignement. Revue des sciences de l'éducation, 28(2), p. 305-324.

Charlier, B., Daele, A. et Deschryver, N. (2002). Vers une approche intégrée des technologies de l'information et de la communication dans les pratiques d'enseignement. Revue des sciences de l'éducation, 28(2), p. 345-365.

Coen, P.-F. (sous presse). Intégrer les TIC dans son enseignement ou changer son enseignement pour intégrer les TIC : une question de formation ou de transformation. Dans B. Charlier et D. Peraya (dir.), Regards croisés sur la recherche en technologies de l'éducation. Bruxelles : De Boeck.

Darling-Hammond, L. (2006). Powerful teacher education. Lessons from exemplary programs. San Francisco : Jossey-Bass.
Haeuw, F. (2002). Technologies en formation et compétences des acteurs: adaptation ou transformation? Éducation permanente, 152, p. 7183.

Isabelle, C., Lapointe, C. et Chiasson, M. (2002). Pour une intégration réussie des TIC à l'école: de la formation des directions à la formation des maîtres. Revue des sciences de l'éducation, 28(2), p. 325-343.

Karsenti. T. et Larose, F. (2005). L'intégration pédagogique des TIC dans le travail enseignant: recherches et pratiques. Québec, Canada : Presses de l’Université du Québec.

Karsenti, T., Peraya, D. et Viens, J. (2002a). Introduction - Formation des enseignants à l'intégration pédagogique des TIC. Esquisse historique des fondements, des recherches et des pratiques. Dans J. Viens, D. Peraya et T. Karsenti (dir.), Intégration pédagogique des TIC: recherches et formation (numéro thématique). Revue des sciences de l'éducation, 28(2), p. 243-264.

Karsenti, T., Peraya, D. et Viens, J. (2002b). Conclusion - Bilan et prospectives de la recherche sur la formation des maîtres à l'intégration pédagogique des TIC. Dans J. Viens, D. Peraya et T. Karsenti (dir.), Intégration pédagogique des TIC: recherches et formation (numéro thématique). Revue des sciences de l'éducation, 28(2), p. $459-470$.

Karsenti, T., Savoie-Zajc, L. et Larose, F. (2001). Les futurs enseignants confrontés aux TIC: changements dans l'attitude, la motivation et les pratiques pédagogiques. Éducation et francophonie, 29(1), p. 1-29.

Larose, F., Grenon, V. et Lafrance, S. (1999). Pratiques et profils d'utilisation des TIC chez les enseignants d'une université. Dans P. Bordeleau, C. Depover et L.-O. Pochon (dir.), L'école de demain à l'heure des technologies de l'information et de la communication. Neuchâtel, Suisse: Institut de 
recherche et de documentation pédagogique (IRDP).

Larose, F. et Karsenti, T. (2002). La place des TIC en formation initiale et continue. Sherbrooke, Canada : Les éditions du Centre de ressources pédagogiques (CRP) de la Faculté d'éducation de l'Université de Sherbrooke.

Larose, F., Lenoir, Y., Karsenti, T. et Grenon, V. (2002). Les facteurs sous-jacents au transfert des compétences informatiques construites par les futurs maîtres du primaire sur le plan de l'intervention éducative. Revue des sciences de l'éducation, 28(2), p. 265-287.

Larose, F., Grenon, V., Pearson, M., Morin, J.-F. et Lenoir, Y. (2004). Les facteurs sociologiques et pédagogiques qui affectent les pratiques des enseignants du primaire au regard de l'informatique scolaire. Dans J.F. Desbiens, J.F. Cardin et D. Martin (dir.), Intégrer les TIC dans l'activité enseignante: Quelle formation? Quels savoirs ? Quelle pédagogie ? (p. 59-80). Québec: Presses de l'université Laval.
Linard, M. (2002). Conception de dispositifs et changement de paradigme en formation. Éducation permanente, 152, p. 143-155.

Mergendoller, J. R., Johnston, J., Rockman, S. et Willis, J. (1994). Case studies of exemplary approaches to training teachers to use technology. Novato, CA : Buck Institute for Education.

OCDE (1998). Compte rendu du séminaire sur les NTIC, Comité de l'Éducation, OCDE. Récupéré du site www.oecd.org/.

Peraya, D. (2002). Communication et nouvelles technologies. In P. Perrig-Chiello et F. Darbellay (dir.), Qu'est-ce que l'interdisciplinarité? Les nouveaux défis de l'enseignement (p. 117-143). Lausanne: Institut universitaire Kurt Bösch.

Perrenoud, P. (1998). Se servir des technologies nouvelles. Genève: Faculté de psychologie et de sciences de l'éducation, Université de Genève.

\section{Notes}

${ }^{1}$ EDUC 2006 représente la suite logique du programme TIC-Jura-2002, qui était un plan d'action pour rendre effective avant 2002 l'entrée des nouvelles technologies de l'information dans les écoles. Son ambition était de préparer les élèves jurassiens de l'école enfantine au secondaire 1 à entrer dans la société de l'information.

Il associe plusieurs services cantonaux et se déroule en partenariat avec les autorités scolaires locales. Sa mission est de définir un concept d'utilisation des TIC pour l'école jurassienne, d'élaborer, coordonner et suivre des projets dans ce domaine, de conseiller et appuyer les écoles et le corps enseignant, de diffuser des informations et de gérer le serveur Web «DUC».

${ }^{2}$ F3-MITIC est une formation de formateurs de formateurs (F3) dans le domaine des médias, de l'image (MI) et des technologies de l'information et de la communication (TIC). 